\title{
THE TEMPORAL STABILITY OF A DEVELOPING JET: A MODEL PROBLEM
}

\author{
JILLIAN A. K. STOTT and JAMES P. DENIER ${ }^{12}$
}

(Received 10 October 1993; revised 28 March 1994)

\begin{abstract}
The temporal instability of a developing swirling incompressible jet is considered. The jet development (in the streamwise direction) is modelled by combining a near-field and farfield approximation to the jet velocity profile into a one parameter family of basic velocity fields. The single parameter in the jet velocity field then allows us to model the radial spreading of the jet and the decay of swirl observed experimentally. Two distinct modes of instability of this model profile are found. The first is that found from a stability analysis of a fully developed swirling jet in the far field whilst the second is relevant to a "top-hat" jet with an imposed rigid body rotation. We demonstrate that the effect of azimuthal swirl is to destabilise both modes of instability. Additionally our results suggest that the near-nozzle modes of instability will dominate; indeed the growth rates of these modes are significantly larger than those found from previous studies of a fully developed jet in the far-field region.
\end{abstract}

\section{Introduction}

Our concern is with the temporal stability of an inviscid swirling jet. We consider a model problem in which the jet velocity field is constructed as a one parameter family of solutions of the Euler equations of motion. This combination is chosen so as to consist of a near-nozzle component and a far-field (a long distance downstream of the jet nozzle) component; the variation of the single parameter appearing in the unperturbed velocity profile then allows us to model the axial variation of the velocity field observed experimentally.

The problem of the instability of jets, and subsequent transition to turbulence, has received considerable attention in recent years due to their importance in the mixing of fuel/air in jet engines of aircraft. Recent work has demonstrated that the addition of a small amount of azimuthal swirl to the jet can increase the linear growth rates

\footnotetext{
'School of Mathematics, The University of NSW, PO Box 1, Kensington, NSW 2033, Australia.

${ }^{2}$ Now at Department of Applied Mathematics, University of Adelaide, SA 5005, Australia.

(C) Australian Mathematical Society, 1995, Serial-fee code 0334-2700/95
} 
of small amplitude disturbances, leading ultimately to enhanced mixing within the jet. This enhanced mixing can greatly increase the overall efficiency of jet engines resulting in a decrease in fuel costs.

These is a considerable body of literature concerning the stability properties of nonswirling jets, but only recently has attention tumed to the problem of the stability of swirling jets. Two distinct approaches to the stability of nonswirling jets have been considered and a variety of basic velocity profiles have been considered. Early work by Batchelor and Gill [1] considered the temporal stability of an asymptotic form of an exact solution of the Navier-Stokes equations; such a solution being relevant to the far-field (a long distance downstream of the jet nozzle) fully developed structure of an axisymmetric jet. Such an instability analysis demonstrates that only the first sinuous mode (that is, that mode with azimuthal wavenumber $m=1$ ) is inviscidly unstable. In contrast, the work of Michalke [10] (and others; see the review article [10] for a full list of relevant publications) is concerned with the spatial instability of a variety of model profiles which are constructed so as to model the jet velocity field in the near-nozzle region. Such studies again demonstrate the instability (now spatial in character) of these (model) velocity profiles.

The extension of these results to the problem of the stability of swirling jets has been considered by a number of authors ([2,4,6-7,13-14]). The extension of the work of Batchelor and Gill [1] to swirling jets has been considered in [4,6-7,13]. In this case, the basic velocity profile for the swirling jet is given by the asymptotic solution found by Loitsyanyskii [9] (see also Görtler [5], Rosenhead [12], pages 452-454) and is a combination of the far-field form of Batchelor and Gill [1] together with an asymptotic form for the azimuthal velocity component. The stability studies of this profile by Khorrami [6-7] and Stott [13] (both within a compressible setting) are posed in terms of a temporal stability analysis. Their results demonstrate that the addition of a small amount of azimuthal swirl destabilises the non-axisymmetric modes with negative azimuthal wavenumber and it is the first non-axisymmetric mode $(m=-1)$ which is the most unstable. When the azimuthal wavenumber is positive, the addition of swirl stabilises these modes (in the sense of decreased growth rates, when compared to the case of zero swirl) and for sufficiently large azimuthal swirl, these disturbances may become completely stabilised.

In the near-nozzle regime, Coleman [2], motivated by astrophysical concerns, considered the temporal stability of a compressible swirling jet. The jet profile employed is a combination of a Rankine vortex superimposed upon a top-hat velocity profile and as such is relevant to the near-nozzle regime. The findings of this work demonstrate that disturbances with positive azimuthal wavenumber are stabilised by small amounts of swirl whereas those with negative azimuthal wavenumbers persist even when the imposed swirl is considerable. However, this work suggests that the magnitude of the growth rates of these modes are substantially reduced with increasing 
swirl. Further work on this near-field profile by Wu et al. [14], who consider the spatial stability, found that when the azimuthal wavenumber is negative the modes are destabilised as the jet rotation is increased, thus contradicting the results of Coleman [2].

The question as to which form of instability is relevant to jets (both swirling and nonswirling) is as yet undecided. The literature, to date, is divided along the lines of near-field profile/spatial stability analysis and far-field profile/temporal stability analysis. The question as to which form of instability is relevant then becomes a question of whether the jet velocity field is absolutely or convectively unstable. To date this question has yet to be answered although some preliminary studies by Foster [4], Lieb and Goldstein [8] and Papageorgiou [11] suggest that such flows are absolutely unstable (at least for a range of system parameters). If such a result were true for a general jet velocity field, it would be the temporal stability of the flow which would be of the most physical relevance. It would then be of interest to consider a temporal stability analysis of a developing jet in order to ascertain whether the nearnozzle modes persist downstream of the nozzle and how, if at all, these modes are related to those modes of instability found in studies of the fully developed profiles valid in the far-field asymptotic limit. Such is the concern of this work.

The rest of this paper is structured as follows. In Section 2 we develop a model velocity profile which incorporates both the, experimentally observed, radial spread and decay of swirl in a real jet. We pose an inviscid, temporal, stability problem for this model profile which is solved by standard numerical techniques. In Section 3 we present our results and in Section 4 we present some conclusions which follow from our work.

\section{Formulation}

We consider the instability of an incompressible swirling round jet. Assuming the jet to be inviscid the governing equations are the Euler and continuity equations in cylindrical polar coordinates. A suitable nondimensionalisation is found by taking the jet nozzle radius as the characteristic length scale and the centerline axial velocity as the characteristic speed. In nondimensional form we then have

$$
\begin{aligned}
& \frac{\partial \mathbf{v}}{\partial t}+\mathbf{v} \cdot \nabla \mathbf{v}=-\nabla p, \\
& \nabla \cdot \mathbf{v}=0,
\end{aligned}
$$

where $\mathbf{v}=(u, v, w)$ is the velocity field referred to cylindrical coordinates $(x, r, \theta)$ (here $x$ is the axial coordinate and $\theta$ is the azimuthal coordinate). We assume the basic flow is steady and of the form

$$
(u, v, w, p)=(\bar{U}(r), 0, \bar{W}(r), \bar{P}(r)),
$$


where $\bar{U}, \bar{W}$ satisfy Euler's equations and $\bar{P}$ is found from the second momentum equation.

Our primary concern in this paper is to model the streamwise variation of the basic flow. In particular, we wish to model two experimentally observed characteristics of swirling jets: (i) radial spreading of the jet as a function of the axial coordinate $x$, (ii) the decay of swirl in the jet as a function of the axial coordinate $x$.

In order to achieve this aim we construct a one parameter family of velocity profiles in the form (2.2), where

$$
(\bar{U}, \bar{W})=(1-b)\left(U_{0}, W_{0}, 0\right)+b\left(U_{1}, W_{1}, 0\right) .
$$

The parameter $b$ in (2.3) represents the axial position $x$ in the flow; however, note that if $b$ were the axial position (2.3) would not satisfy the Euler equations. The specific choice of profiles $U_{0}, U_{1}$ and $W_{0}, W_{1}$ appearing in (2.3) is effected by comparison with experimentally measured velocity profiles. The work of $\mathrm{Wu}$ et al. [14] demonstrates that near the jet nozzle the axial velocity closely resembles the "plug-flow" (or top-hat) jet profile induced by a cylindrical vortex sheet while the azimuthal velocity resembles that obtained from a rigid-body rotation within the nozzle radius. The far-field (in this case several nozzle radii downstream of the jet nozzle) velocity field closely resembles the similarity form found by Loitsyanskii [9] (see also Görtler [5] and Rosenhead [12] pages 452-454). With these results in mind we define

$$
\begin{array}{ll}
U_{0}=\frac{1}{2}[1+\tanh (\tilde{c}-\tilde{c} r)], & W_{0}=\sigma r U_{0}, \\
U_{1}=\frac{1}{\left(1+r^{2}\right)^{2}}, & W_{1}=\sigma r U_{1} .
\end{array}
$$

Here we note that the profile $U_{0}$ corresponds to that used by Michalke [10]; in the limit $\tilde{c} \rightarrow \infty$ it reduces to the "plug-flow" velocity profile and as such approximates the near nozzle axial velocity profiles measured experimentally. The addition of the azimuthal component $W_{0}$ then gives a good approximation to the near nozzle azimuthal velocity found in experiments. The second component of the basic velocity field $\left(U_{1}, W_{1}\right)$ corresponds to the asymptotic similarity solution found by Görtler [5]. We can now see the relevance of the parameter $b$ in our basic flow (2.3). Setting $b=0$ reduces (2.3) to $\bar{U}=U_{0}$ while for $b=1$ we have $\bar{U}=U_{1}$. Thus varying the parameter $b$ between zero and one allows us to model the effect of the streamwise variation of the jet velocity field from a near-field form to the far-field similarity form. In Figure la we present a plot of $\bar{U}$ given by (2.3) as a function of $b$ (for the particular choice $\tilde{c}=6$ ) from which we may readily observe the radial spreading of the jet as a function of $b$. We note at this point that throughout this work we will choose, for definiteness, the parameter value $\tilde{c}=6$; other larger values of this parameter were considered, however its precise value had little effect on our subsequent stability calculations. 
In order to model the decay of swirl found experimentally we turn our attention to the azimuthal component of the velocity field. In $(2.4 \mathrm{a}, \mathrm{b})$, the parameter $\sigma$ is a measure of the mean flow angularity. Previous studies into swirling jets by Khorrami [6] and Stott [13] have demonstrated that the stability of swirling jets is dependent on the swirl ratio $q$, which is defined as the ratio of the maximum azimuthal velocity to the maximum axial velocity; note that the maximum axial velocity given by (2.3) has been normalised to be unity. The parameter $\sigma$ in (2.4) is then chosen so that the swirl ratio at $b=1$, (that is, in terms of our model profile, this corresponds to the far field similarity solution of Görtler [5], which is the basis of the studies by Khorrami [6] and Stott [13]) is fixed at some prescribed value, $q_{1}$ say. Thus

$$
\sigma=q_{1} / \max \left(r U_{1}\right) .
$$

A plot of $q$ versus $b$ is given in Figure 2, which together with a plot of $\bar{W}$ versus $r$ at various values of the parameter $b$, given in Figure $1 \mathrm{~b}$, demonstrates that our model profile (2.3) adequately models the decay of swirl as a function of the axial coordinate.
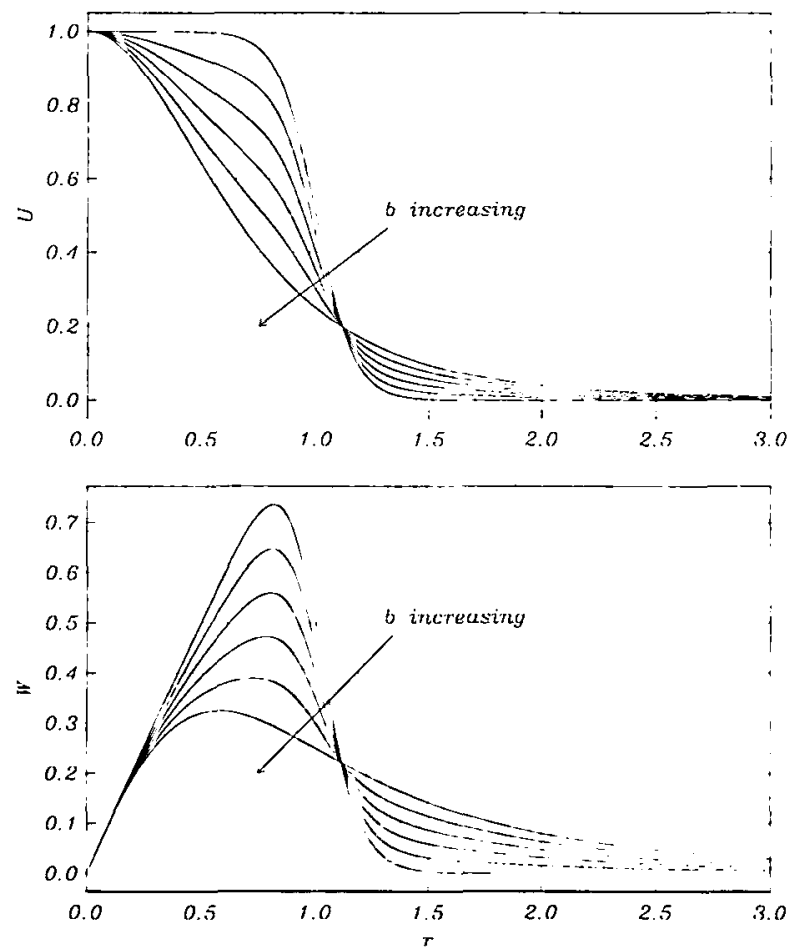

FIGURE 1. A plot of the jet velocity profile for various values of the parameter $b$; (a) the radial velocity, (b) the azimuthal velocity. 


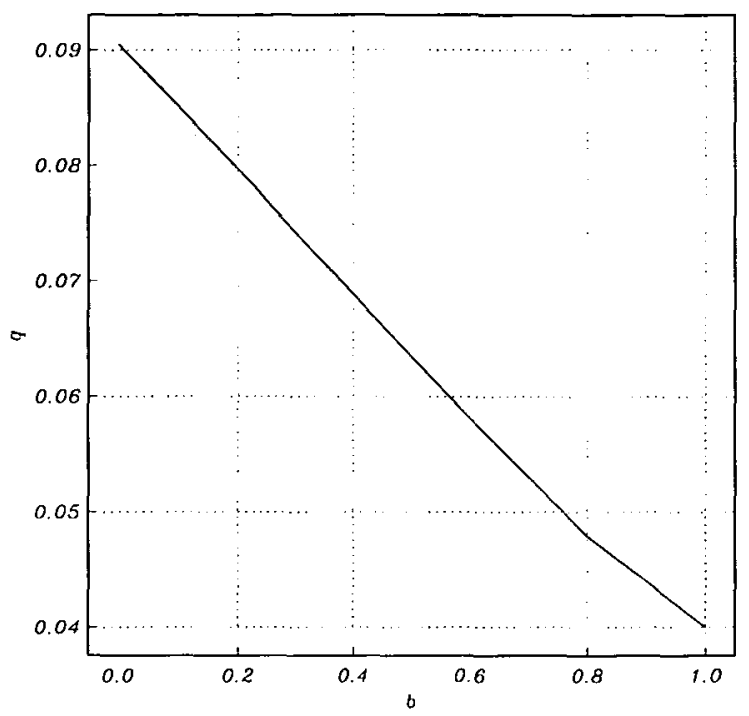

FlGURE 2. A plot of the swirl parameter $q$ as a function of $b$.

To proceed we take the basic flow to be given by (2.3) and consider perturbations to this flow which satisfy the nondimensionalised Euler and continuity equations in cylindrical coordinates. Consider then perturbations in the form of travelling waves disturbances

$$
\mathbf{v}=\overline{\mathbf{v}}+\delta \mathbf{V} E, \quad p=\bar{P}+\delta P E,
$$

where

$$
E=\exp [i(\alpha x+m \theta-\omega t)],
$$

and $\delta \ll 1$ is a small amplitude parameter. Substitution of the expansion (2.5) into (2.1) and linearizing with respect to the unperturbed flow gives the following system of equations for the perturbation quantities

$$
\begin{aligned}
\Omega U+V \frac{d \bar{U}}{d r}+\alpha P & =0, & -\Omega V-\frac{2 \bar{W}}{r} W+\frac{d P}{d r}=0, \\
\left(\frac{d \bar{W}}{d r}+\frac{\bar{W}}{r}\right) V+\Omega W+\frac{m}{r} P & =0, & \alpha U+\frac{V}{r}+\frac{d V}{d r}+\frac{m W}{r}=0,
\end{aligned}
$$

where $\Omega$ is the group parameter given by $\Omega=\alpha \bar{U}-\omega+m \bar{W} / r$. Eliminating $U$ and $W$ from (2.6) then gives

$$
\frac{d V}{d r}=\left(\frac{\alpha r^{2} \bar{U}^{\prime}+m(r \bar{W})^{\prime}}{\Omega r^{2}}-\frac{1}{r}\right) V+\frac{\left(\alpha^{2} r^{2}+m^{2}\right)}{\Omega r^{2}} P
$$




$$
\frac{d P}{d r}=\left(\Omega-\frac{2 \bar{W} r(r \bar{W})^{\prime}}{\Omega r^{3}}\right) V-\frac{2 m \bar{W}}{\Omega r^{2}} P,
$$

where primes denote differentiation with respect to $r$. The boundary conditions appropriate to the disturbances quantities are found by modifying the arguments presented by Batchelor and Gill [1], to account for the presence of the azimuthal velocity component; in terms of $P$ and $V$ we have

$$
\begin{aligned}
& P(0)=0, \quad m \neq 0 ; \quad P^{\prime}(0)=0, \quad m=0, \\
& V(0)=0, \quad|m| \neq 1 ; \quad V^{\prime}(0)=0, \quad|m|=1, \\
& V(r), P(r) \rightarrow 0 \text { as } r \rightarrow \infty \text {. }
\end{aligned}
$$

The differential equations (2.7) together with the boundary conditions (2.8) then constitute an eigenvalue problem for $\omega=\omega(\alpha, m, q, b)$, in the case of a temporal stability analysis, or $\alpha=\alpha(\omega, m, q, b)$, in the case of a spatial stability analysis. Previous results suggest that a spatial stability analysis is appropriate to the near nozzle region, (in our model $b \ll 1$ ), whilst a temporal stability analysis is appropriate to the far field region, (in our model this corresponds to $b \approx 1$ ). However, the question as to whether the instability of a free jet is convective or absolute is still to be settled, although recent results by Leib and Goldstein [8], Foster [4] and Papageorgiou [11] suggest that the flow is absolutely unstable. If, indeed, such a result is generic the temporal eigenvalue problem would be the most relevant to the problem of swirling free jets. For definiteness, we will restrict our attention to the problem of temporal stability (it is anticipated that the question as to the spatial stability of the basic flow (2.2) will be considered in a future paper).

Thus, the eigenvalue problem posed by $(2.7,8)$ will be considered in the following form: given a real wavenumber $\alpha$ determine the complex wavespeed $\omega=\alpha c=$ $\alpha\left(c_{r}+i c_{i}\right)$ as a function of the swirl ratio $q$, the variational parameter $b$ and the azimuthal wavenumber $m$.

Eliminating $V$ from (2.7) yields a single second order ordinary differential equation for the pressure perturbation $P$ in the form

$$
C P^{\prime \prime}-\left[(D+A) C+C^{\prime}\right] P^{\prime}+\left[C^{\prime} D-C D^{\prime}+A C D-B C^{2}\right] P=0,
$$

where

$$
\begin{aligned}
A & =\frac{\alpha r^{2} \bar{U}^{\prime}+m(r \bar{W})^{\prime}}{\Omega r^{2}}-\frac{1}{r}, & B & =\frac{\alpha^{2} r^{2}+m^{2}}{\Omega r^{2}}, \\
C & =\Omega-\frac{2 \bar{W} r(r \bar{W})^{\prime}}{\Omega r^{3}}, & D & =-\frac{2 m \bar{W}}{\Omega r^{2}},
\end{aligned}
$$

and the primes denote differentiation with respect to the radial coordinate $r$. 
A fourth order Runge-Kutta scheme is used to numerically integrate the second order differential equation (2.9) coupled with a standard Newton iteration technique to determine the eigenvalues. In order to take care of the singularity at $r=0$ appearing in (2.9), we make use of the Frobenius expansion of the solution of (2.9) at $r=0$,

$$
P=P_{0} r^{|m|}+\text { lower order terms } \quad \text { as } \quad r \rightarrow 0,
$$

where we have chosen the solution which remains bounded as $r \rightarrow 0$. (We note here that the leading order behaviour of $P$ as $r \rightarrow 0$ is found to be $P \sim r^{ \pm m}$ and thus to ensure boundedness of $P$ as $r \rightarrow 0$ we choose $P \sim r^{m}$ for $m>0$ and $P \sim r^{-m}$ for $m<0$ ). In the limit $r \rightarrow \infty$, we impose the asymptotic boundary condition

$$
P \sim A e^{-\alpha r}+\text { lower order terms. }
$$

Since the system (2.9) is linear in $P$, we are free to normalise $P$ such that

$$
P=r^{|m|}+\text { lower order terms } \quad \text { as } \quad r \rightarrow 0 .
$$

This normalisation was chosen solely for convenience; the precise form of the normalization at $r=0$ has no effect upon the eigenvalues found for (2.9).

The system (2.9) subject to (2.10) and (2.11) (where (2.10) is imposed at some suitably large value of $r=r_{\infty}$ ) then defines our eigenvalue problem for which the complex wavespeed $c=c_{r}+i c_{i}$ and the complex amplitude $A$ are to be determined. An initial guess is made for $c$ and $A$ and (2.9) is integrated from $r=r_{\infty}$ to some fixed point $r=r_{m}$ (typically $r_{m}=r_{\infty} / 2$ in all the calculations reported here). In addition the system (2.9), subject to the initial condition (2.11), imposed at some suitably small value of $r=\Delta r$ (where $\Delta r$ is the step-size used in the Runge-Kutta integration), is integrated to $r=r_{m}$. Newton iteration is then employed on the unknowns $c$ and $A$ until matching of $P$ and $P_{r}$ at $r=r_{m}$ is achieved (to within some desired tolerance).

In all the calculations reported here $r_{\infty}$ was chosen so as to give a suitable decay at $r=r_{\infty}$ and the step-size $\Delta r$ was reduced until the change in the calculated values of $c$ and $A$ was negligible. In Table 1 we give some representative, convergence results for the eigenvalues $c_{r}+i c_{i}$ as a function of the number of grid points and the position $r_{\infty}$ (for the particular choice of parameters $\alpha=1.0, b=0.0$ and $m=0$ ) from which we observe that for $r_{\infty}=10.0$ and $N=2000$ the eigenvalues have converged to within graphical accuracy. Thus, in all that follows we will take $N=2000$ and a maximum value of $r_{\infty}=10.0$ (the precise value of $r_{\infty}$ used depends upon the wavenumber $\alpha$; for large wavenumbers a smaller value of $r_{\infty}$ is appropriate in light of the exponential decay seen in (2.10)). 
TABLE 1.

\begin{tabular}{|c|c|c|c|c|}
\hline \multicolumn{5}{|c|}{${ }^{\dagger}$ Convergence of eigenvalues as a function of $r_{\infty}$} \\
\hline$r_{\infty}$ & $N$ & $c_{r}$ & $c_{i}$ & $\left.\left(c_{i}-c_{i}^{*}\right) / c_{i}^{*}\right)$ \\
\hline 10.0 & 2000 & 0.69657890 & 0.38279068 & $0.20951398(-04)$ \\
\hline 11.0 & & 0.69657936 & 0.38279012 & $0.19488486(-04)$ \\
\hline 12.0 & & 0.69657986 & 0.38278952 & $0.17921076(-04)$ \\
\hline 13.0 & & 0.69658041 & 0.38278885 & $0.16170795(-04)$ \\
\hline 14.0 & & 0.69658101 & 0.38278813 & $0.14289889(-04)$ \\
\hline 15.0 & & 0.69658166 & 0.38278736 & $0.12278357(-04)$ \\
\hline 16.0 & & 0.69658235 & 0.38278653 & $0.10110074(-04)$ \\
\hline 17.0 & & 0.69658310 & 0.38278565 & $0.7811601(-05)$ \\
\hline 18.0 & & 0.69658390 & 0.38278471 & $0.53554908(-05)$ \\
\hline 19.0 & & 0.69658475 & 0.38278371 & $0.27430634(-05)$ \\
\hline 20.0 & & 0.69658565 & $0.38278266^{*}$ & \\
\hline \multicolumn{5}{|c}{${ }^{\dagger}$ Convergence of eigenvalues as a function of $N$} \\
\hline$r_{\infty}$ & $N$ & \multicolumn{4}{|c|}{$c_{r}$} & $c_{i}$ & $\left(c_{i}-c_{i}^{*}\right) / c_{i}^{*}$ \\
\hline 10.0 & 1000 & 0.69628713 & 0.38249592 & $-0.27561332(-04)$ \\
\hline & 2000 & 0.69657890 & 0.38279068 & $-0.66093563(-05)$ \\
\hline & 3000 & 0.69657769 & 0.38279215 & $-0.27691268(-05)$ \\
\hline & 4000 & 0.69657728 & 0.38279266 & $-0.14368092(-05)$ \\
\hline & 5000 & 0.69657708 & 0.38279290 & $-0.80983738(-06)$ \\
\hline & 6000 & 0.69657698 & 0.38279302 & $-0.49635179(-06)$ \\
\hline & 7000 & 0.69657692 & 0.38279310 & $-0.28736150(-06)$ \\
\hline & 8000 & 0.69657688 & 0.38279315 & $-0.15674262(-06)$ \\
\hline & 9000 & 0.69657685 & 0.38279319 & $-0.52247533(-07)$ \\
\hline & 10000 & 0.69657683 & $0.38279321^{*}$ & \\
\hline & &
\end{tabular}

${ }^{\dagger}$ (all results for $\alpha=1.0, b=0.0, r_{m}=r_{\infty} / 2, m=0$ )

\section{Results}

Throughout we will, in order to reduce the number of free parameters in the problem, restrict our attention to the case when the parameter $\tilde{c}$, appearing in (2.4), is fixed at $\tilde{c}=6$. This value was chosen solely for convenience and is in no way of special significance. The results found for larger values of $\tilde{c}$ are qualitatively similar to those to be reported here, with the general trend that increasing $\tilde{c}$ causes a corresponding increase in the growth rate. 

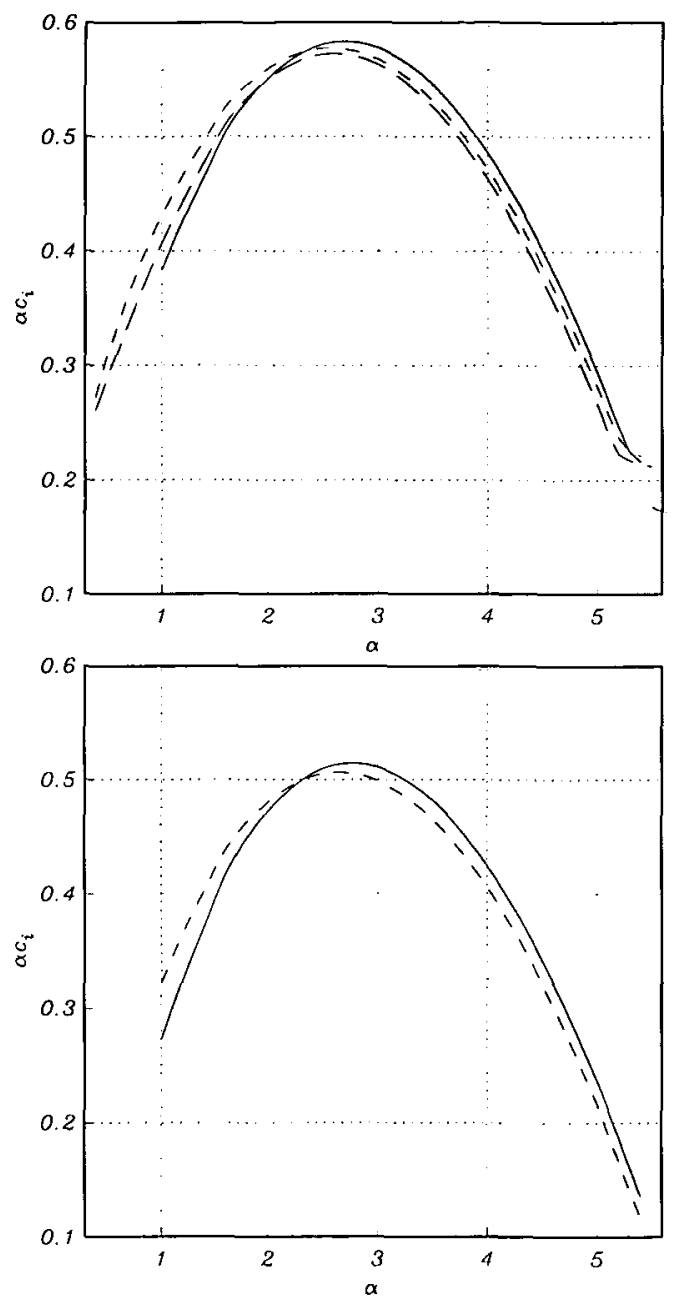

FIGURE 3. A plot of the growth rates of the most unstable mode as a function of $\alpha$ for $b=0$; (a) $q_{1}=0.04, m=0(-), m=1(--), m=-1(--)$, (b) $q_{1}=0, m=0(-),|m|=1(--)$.

Firstly we examined the growth rates of the modes when $b=0$, that is, in the near-nozzle region. Spatial stability studies have been carried out on this profile for nonswirling jets (see Michalke [10]). Swirling jets in the near-nozzle region have been examined by Coleman [2] who considered the temporal stability of such flows and Wu et al. [14] who studied the spatial stability problem. In both cases the flow considered was a combination of a "top-hat" jet with an imposed Rankine vortex. Coleman [2] found that for positive azimuthal wavenumbers the introduction of a small amount of swirl temporally stabilises all modes. Wu et al. [14] also obtained this result for the 
spatial stability problem; in addition they found that when the azimuthal wavenumber is negative, the introduction of swirl spatially destabilises modes. However the work of Coleman [2] suggests, when considering temporal stability theory, that although modes persist when the azimuthal wavenumber is negative even when jet rotation is considerable, the growth rates of these modes is substantially reduced.

Figure 3(a) and 3(b) show plots of the growth rates of the most unstable mode versus the axial wavenumber $\alpha$ with swirl $\left(q_{1}=0.04\right)$ and no swirl respectively, for the near nozzle profile described in (2.4a). From these figures we can see that the inclusion of swirl in the jet has a temporally destabilising effect on the modes $(m=-1,0,1)$ in this near-nozzle region, which contradicts the results found by Coleman [2].

TABLE 2. Maximum growth rate as a function of azimuthal wavenumber $m$. Here $b=0$ with swirl ratio $q_{1}=0.04$. The terms in the braces correspond to the zero swirl case.

\begin{tabular}{|c|cc|cc|}
\hline \multicolumn{3}{|c|}{${ }^{\dagger}$ Maximum growth rates $\alpha c_{i}$} \\
\hline$m$ & \multicolumn{2}{|c|}{$\alpha$} & \multicolumn{2}{|c|}{$\alpha c_{i}$} \\
\hline-4 & 2.0 & 0.3327 & \\
\hline-3 & 2.5 & 0.4160 & \\
\hline-2 & 2.6 & & 0.5109 & \\
\hline-1 & 2.6 & & 0.5773 & \\
\hline 0 & 2.7 & $(3.0)$ & 0.5830 & $(0.5113)$ \\
\hline 1 & 2.6 & $(2.6)$ & 0.5723 & $(0.5064)$ \\
\hline 2 & 2.6 & $(2.7)$ & 0.4949 & $(0.4309)$ \\
\hline 3 & 2.5 & $(2.7)$ & 0.3766 & $(0.3153)$ \\
\hline 4 & 2.2 & $(2.4)$ & 0.2468 & $(0.1900)$ \\
\hline
\end{tabular}

${ }^{\dagger}$ All results for $b=0, q_{1}=0.04$.

In Table 2, we present values of the maximum growth rate as a function of the azimuthal wavenumber from which we readily see a decrease in the maximum growth rate as $|m|$ increases.

The effect of swirl may also be clearly seen in Figure 4, where growth rate curves for the most unstable modes are displayed with $b=0$ for Figures (a)-(c) and $b=0.5$ for (d)-(f). For each plot, $q_{1}$ is progressively increased from 0 to 0.1 in increments of 0.02 . It can be seen that when $b=0.5$, the behaviour of the flow with respect to the parameter $q_{1}$ is similar to the behaviour when $b=0$; however the flow appears to have become less unstable with its progression downstream.

In Figure 5, the eigenfunctions for the most unstable modes, for the case $b=0$, are shown. Three different values of $m$ are displayed and for each plot the axial wavenumber is increased at regular intervals. From these figures, it is apparent that 

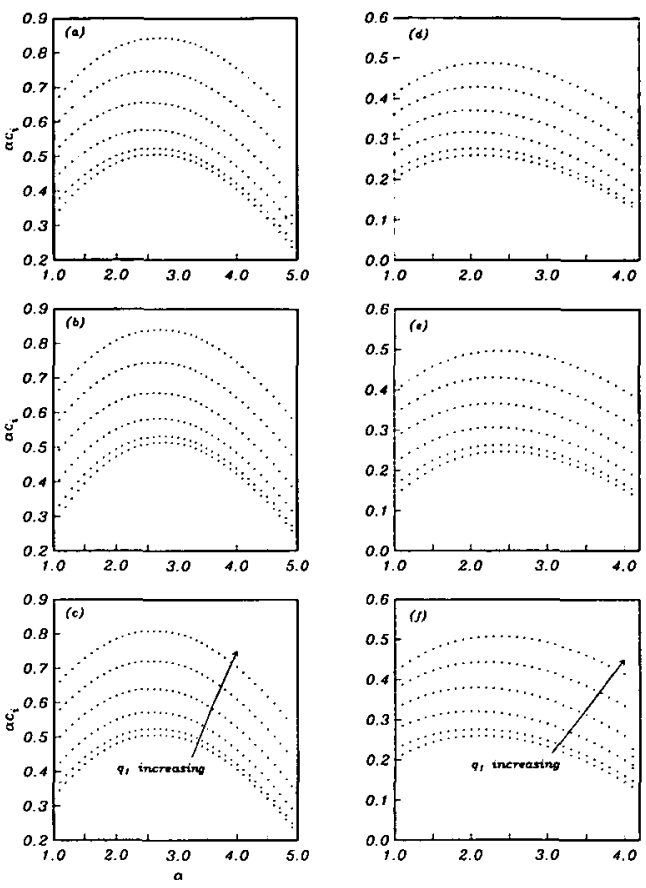

FIGURE 4. A plot of the growth rates of the most unstable mode as a function of $\alpha$ for $q_{1}=$ $0.0,0.02,0.04,0.06,0.08,0.1$ with (a) $m=1, b=0$, (b) $m=0, b=0$, (c) $m=-1, b=0$, (d) $m=1$, $b=0.5$, (e) $m=0, b=0.5$, (f) $m=-1, b=0.5$.

the modes, when $b=0$, become localised about some point $r=r_{0}$, say, a finite distance away from the centreline of the flow. Behaviour of this kind is indicative of a ring mode structure; see Duck [3] for a description of the asymptotic structure of these modes. Indeed, this behaviour was observed at all values of the parameter $b$ in the range $0 \leq b \leq 1$. A structure of this type is also found in the fully-developed jet profile (see Khorrami [6]). We note that, in all cases considered, the value of $r_{0}$, for the near-nozzle modes, is approximately equal to unity and thus is invariant to changes in the parameters $m$ and $\alpha$.

We now turn our attention to Figure 6, which shows the growth rates of a number of different modes obtained when $b=0, m=-1$ and $q=0.04$. These modes are classified, following Michalke [10], as regular or irregular according to whether $\alpha c_{i}$ tends to zero or a nonzero value as $\alpha \rightarrow 0$, respectively. The irregular modes (dashed lines in Figure 6) are found to have significantly smaller growth rates compared to the regular modes; we note that a family of regular modes also exists but the growth rates of these higher order eigenvalues are much smaller than those of the first regular mode (and also the first two irregular modes) and are localised in a small neighbourhood 

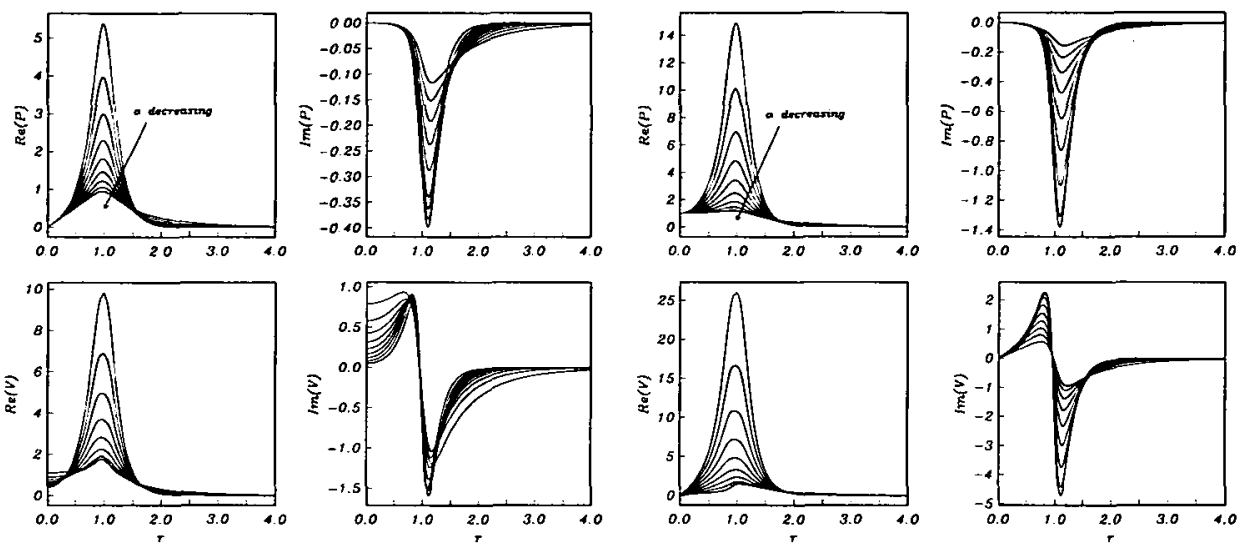

(a)

(b)
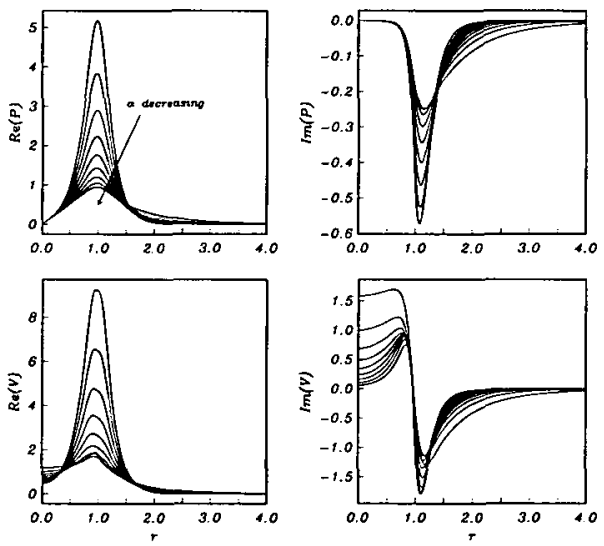

(c)

FIGURE 5. Plots of the eigenfunctions for the most unstable mode for $b=0, q_{1}=0.04$ and $\alpha=1.0,1.5, \ldots, 4.5$ with (a) $m=-1$, (b) $m=0$, (c) $m=1$.

about $\alpha=0$. Due to the size of the growth rates of these modes an accurate determination of their growth rates is difficult; for this reason we do not present plots of them here. We note that the irregular modes of instability have previously been obtained for a nonswirling jet in the near-nozzle region using a spatial stability investigations (see Michalke [10]), however this study appears to be the first which has demonstrated their existence in a temporal setting. The physical significance of the irregular modes is as yet unknown, however it would appear that, judging from their 
small growth rates, they will be dominated by the first regular mode in any evolving, swirling jet. Also, although not displayed here, it is possible to obtain irregular modes of a similar type for all other nonzero values of $m$ provided the swirl ratio is nonzero. In the case $q_{1}=0$ (and arbitrary $m$ ) we were unable to locate any irregular modes. The fact that such modes do not exist when $q_{1}=0$ is also suggested by the fact that the regular modes do not jump as the upper neutral point is approached. This is unlike the case when $q_{1}=0.04$, compared to Figures $3 \mathrm{a}$ and $\mathrm{b}$.

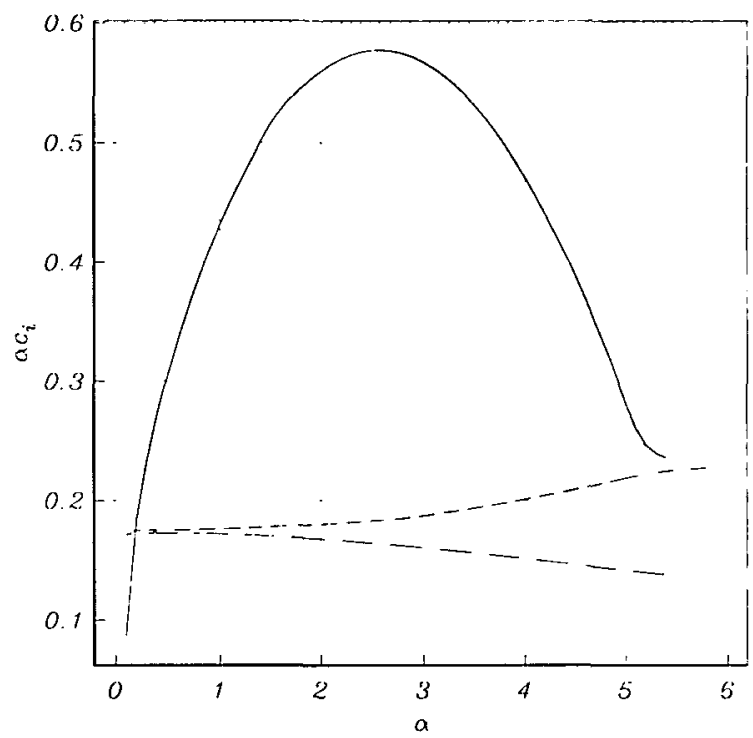

FIGURE 6. Plots of the growth rates of the most unstable regular mode ( - ) and two irregular modes $(--)$ when $b=0$ and $m=-1$.

In Figure 7, we show the eigenfunctions of the most unstable irregular mode shown in Figure 6, for various values of $\alpha$. Figure 7 demonstrates that, as in the case of the regular modes, the eigenfunctions are localised about $r \approx 1$. Hence these irregular modes can also be classified as ring modes. We note that the eigenfunctions presented in Figures 5 and 7 undergo rapid variation in the vicinity of $r=1$. Further examination of the structure of the governing equations (2.7) demonstrates that this rapid variation occurs where $|\Omega|$ ( $\Omega$ being the group parameter defined in Section 2$)$ attains its minimum value.

We now turn our attention to the behaviour of the modes with progression downstream, here modelled by varying the parameter $b$ between zero and unity. This behaviour is modelled in Figures 8 (a)-(d) by surface plots of growth rate, $\alpha c_{i}$, versus $\alpha$ and $b$. By examining Figures 8(a) and 8(b), we see that two separate and distinct 

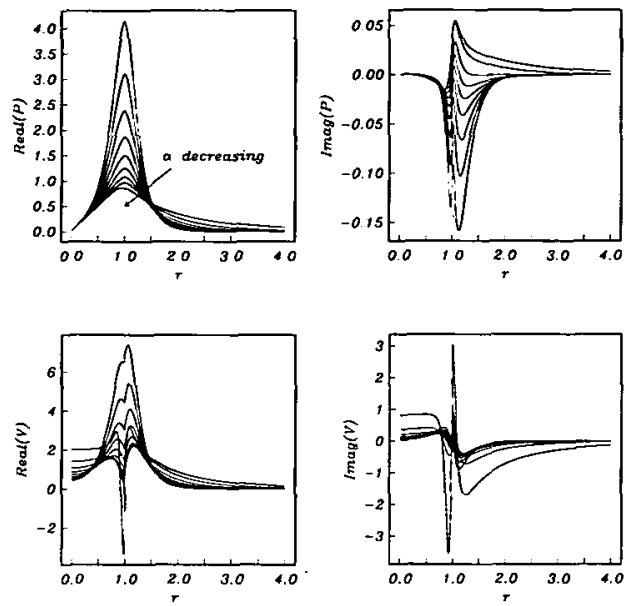

FIGURE 7. Plots of eigenfunctions for the first irregular mode when $b=0$ and $m=-1$ and with varying $\alpha$.

modes are obtained; (i) a mode which emanates at a finite distance downstream from the nozzle of the jet and then becomes increasingly unstable until it attains a maximum value far-downstream (Figure 8a), and (ii) a mode in the near nozzle region of the jet which decays with the progression of the flow downstream (Figure $8 \mathrm{~b}$ ). For the remainder of this paper these modes will be referred to as modes of type I and II respectively.

Figure 8(a) shows a surface plot of the development of the growth rates of the most unstable type I mode against $\alpha$ and $b$ when $m=-1$ and $q_{1}=0.04$. When the jet is fully-developed in the far-downstream region of the flow, the results obtained here agree with those found by Khorrami [6] and Stott [13]. Plots similar to Figure 8(a) can be obtained when $m=0$ and $m=-1$, but as noted by Khorrami [6], the effect of swirl on these modes greatly reduces the magnitude of the growth rates and hence determination of such modes by the technique described in Section 2 proved difficult due to frequent occurrence of the phenomena of mode jumping.

Type II modes are those found in the stability analysis of a "top-hat" jet with an imposed rigid body rotation. Figure 8(b)-(d) show surface plots of the growth rates of the most unstable modes (type II) against $b$ and $\alpha$, for $m=-1,0$ and 1 respectively and in each case $q_{1}=0.04$. It may be seen from these figures that type II modes decay as $b$ increases and eventually become stable at some finite distance downstream. The particular location of the point (in terms of the streamwise parameter $b$ ) at which this occurs is dependent on the axial and azimuthal wavenumber. The precise location at which such modes become stable can be achieved by modifying our numerical 
scheme to deal with the critical layer encountered when the eigenvalue passes through the point of marginal stability. As our main concern is with the instability of the basic flow we do not consider the problem of neutrally stable eigenvalues here.
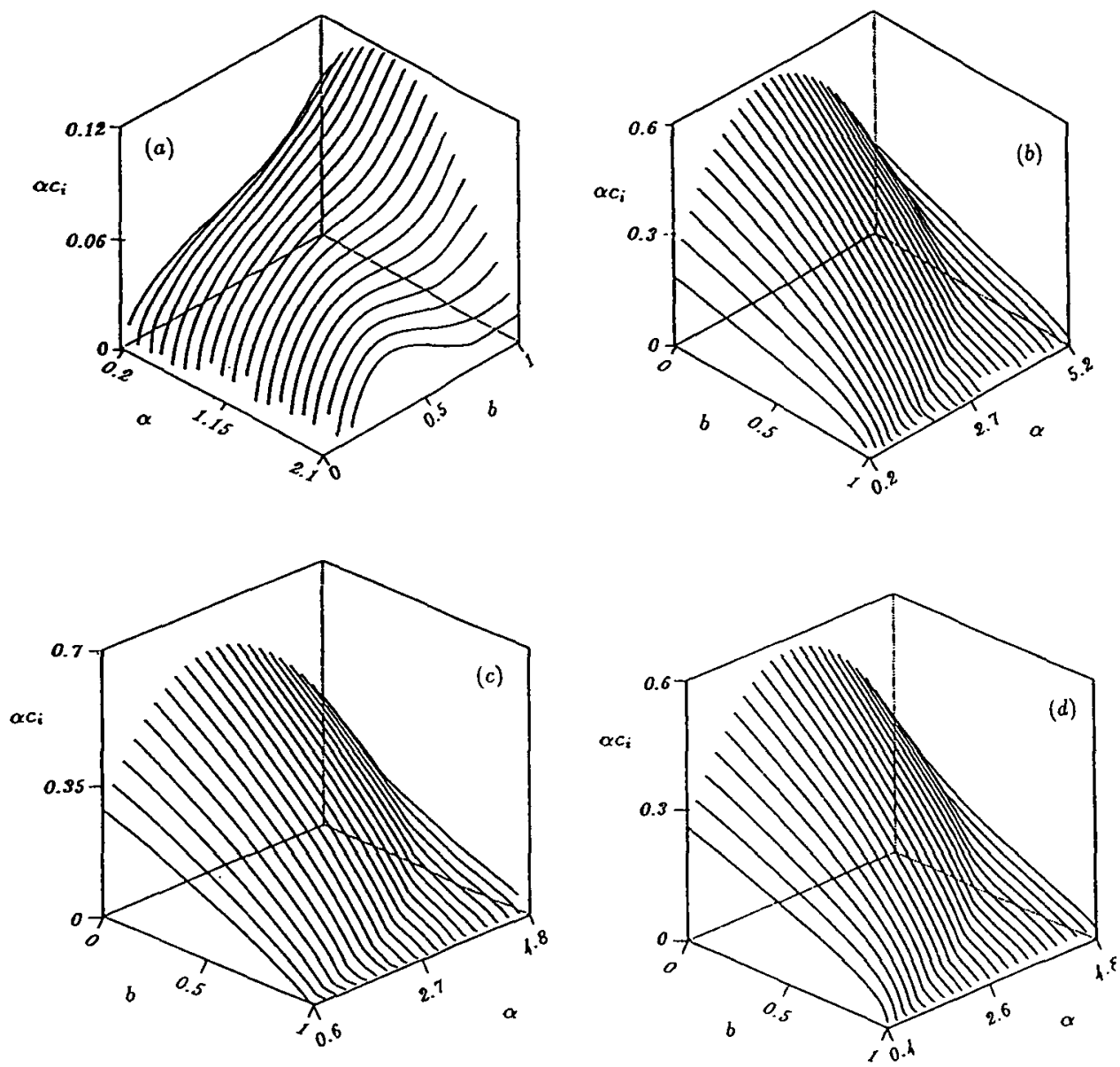

FIGURE 8. A surface plot of the growth rates of the most unstable modes versus $\alpha$ and $b$ for (a) $m=-1$, mode I.

We also find from Figures 8(a) and 8(b) that the magnitudes of the growth rates of modes of type II at $b=0$ are much greater than those found for the fully-developed jet far-downstream, where the type I modes reach their maximum value. This result therefore suggests that modes in the near-nozzle region of the flow are the dominant modes when the temporal stability of such flows is considered. Such a dominant instability in the near-nozzle region would suggest that the far-field structure may never be observed since at such large distances downstream of the jet orifice the initial 
instabilities appearing in the near field may well have rendered the flow fully turbulent.

\section{Conclusions}

We have presented a model problem in which the temporal stability of a swirling jet is considered. The axial variation of the jet is modelled using a one parameter family of jet velocity fields in which the free parameter represents the axial distance from the jet nozzle. Our results demonstrate that there are two distinct modes of instability; each relevant to a different asymptotic regime within the flow. In particular, this study demonstrates that the modes considered by previous authors in their study of the far downstream asymptotic structure of a swirling jet are not, in fact, the dominant mode of instability. The dominant mode is that found to occur in the near-nozzle regime. Such a result would then suggest that the far-field asymptotic structure considered by previous authors is irrelevant in that the unstable modes present in the near field regime (the type II modes discussed above) would be likely to render the jet fully turbulent well before the far field asymptotic velocity field profile is attained.

Several important questions pertaining to the stability of jets, both swirling and nonswirling, remain to be answered. The model problem considered in this work goes one step towards settling the question as to whether the near-nozzle modes of instability are, indeed the dominant ones. Further work is called for to fully answer this question. To do so would require the ideas presented in this work to be put on a firmer mathematical footing by solving the full, nonlinear governing equations (possibly within a boundary layer context), together with some suitable "initial" conditions in the vicinity of the nozzle, to determine the mathematically correct basic flow and to then consider the inviscid instability of such a profile. It is hoped that the results of such a study can be presented in the near future.

\section{Acknowledgements}

This work was supported by the Australian Research Council Small Grants Scheme. We would like to thank an anonymous referee whose comments greatly improved the presentation of this manuscript.

\section{References}

[1] G. K. Batchelor and A. E. Gill, "Analysis of the stability of axisymmetric jets", J. Fluid Mech. 14 (1962) 529-551.

[2] C. S. Coleman, "The stability of swirling jets", Astro. Soc. Aus. Proc. 8 (1989) 38.

[3] P. W. Duck, "The inviscid stability of a swirling flow: large wavenumber disturbance", J. Appl. Math. Phys. (ZAMP) 37 (1986) 340-360. 
[4] M. R. Foster, "Nonaxisymmetric instability in slowly-swirling jet flows" (1993) preprint.

[5] H. Görler, "Theoretical investigations of laminar boundary-layer problems II-theory of swirl in an axially symmetric jet, far from the orifice", Air Force Contract No. AF 61(514) 627C (1954).

[6] M. R. Khorrami, "Stability of a compressible swirling jet", AIAA Paper No 91-1770 (1991).

[7] M. R. Khorrami, "The effect of swirl on the stability of a compressible axisymmetric jet", in Proc. 11th Australasian Fluid Mechanics Conf., 14-18 Dec. 1992, Hobart, Australia (1992) 1101-1104.

[8] S. J. Leib and M. E. Goldstein, "The generation of capillary instabilities on a liquid jet", J. Fluid Mech. 168 (1986) 479-500.

[9] L. G. Loitsyanskii, "Propogation of a rotating jet in an infinite space surrounded by the same liquid", Prik. Mat. Mehk. 17 (1953) 3-16, (in Russian).

[10] A. Michalke, "Survey on jet instability theory", Prog. in Aerospace Sci. 21 (1984) 159-199.

[11] D. T. Papageorgiou, "Stability of cylindrical vortex sheets with swirl and surface tension" (1993) preprint.

[12] L. Rosenhead, Laminar boundary layers (Dover, New York, 1963).

[13] J. A. K. Stott, The stability of compressible swirling flows, Ph. D. Thesis, University of Manchester, 1993.

[14] C. Wu, S. Farokhi and R. Taghavi, "Spatial instability of a swirling jet-theory and experiment", AIAA 30 (1992) 1545-1552. 